\title{
Estudo comparativo dos dados de precipitação do satélite TRMM e postos pluviométricos no estado do Ceará, Brasil
}

\author{
Comparative study of precipitation data from the TRMM \\ satellite and pluviometric stations in the state of Ceará, Brazil
}

Estudio comparativo de los datos de precipitación del satélite
TRMM y postos pluviométricos en el estado del Ceará, Brasil

\author{
Jose Reginaldo Medeiros-Feitosa ${ }^{l}$ \\ Universidade Federal do Cariri, Crato, Ceará, Brasil \\ Carlos Wagner Oliveira ${ }^{2}$ \\ Universidade Federal do Cariri, Crato, Ceará, Brasil
}

\begin{abstract}
Resumo
O Nordeste do Brasil, é caracterizado pelo clima semiárido e pela irregularidade das chuvas ao longo do tempo, que comprometem a agricultura, o abastecimento público e outros setores. Neste sentido, pesquisas que promovam o Desenvolvimento Sustentável dão subsídios para a implementação de novas políticas públicas voltadas para a sustentabilidade hídrica da região. Esta pesquisa teve por objetivo, o de comparar dados de chuva das estações pluviométricas da Fundação Cearense de Meteorologia e Recursos Hídricos (FUNCEME), com as estimativas de precipitação do satélite Tropical Rainfall Measuring Mission (TRMM), entre 1 de janeiro de 1998 a 31 de dezembro de 2017, totalizando vinte anos de análises para todo o estado do Ceará, Brasil. Na primeira parte a pesquisa utilizou as técnicas de comparação do Pixel a Ponto (A e B), Ponto a Pixel (C), e Pixel a Pixel (D), juntamente com o auxílio do interpolador Inverso do Peso da Distância (IDW), na segunda etapa os dados foram analisados por medidas métricas de acurácia: Erro Médio Absoluto
\end{abstract}

1 Maestría en Desarrollo Regional Sostenible - PRODER, Universidad Federal de Cariri - UFCA. Correo electrónico: regincrato@gmail.com. https://orcid.org/0000-0002-7902-5336

2 Orientador, Engenheiro Agrônomo, PhD. Biosystems Engineering, Professor Adjunto no Centro de Ciências Agrárias e da Biodiversidade da Universidade Federal do Cariri - UFCA, correio eletrônico: carlos. oliveira@ufca.edu.br. https://orcid.org/0000-0003-1013-2974 
(EMA), Raiz do Erro Médio Quadrático (REMQ) e o Coeficiente de correlação (r). Os resultados das análises, indicaram que a utilização das imagens do satélite TRMM apresentou-se como boa alternativa com o EMA de 16,46mm na média, o REMQ de $26,78 \mathrm{~mm}$ e a correlação de 0,96 .

Palavras-chave: Recursos Hídricos, Pluviometria, Sensoriamento Remoto.

\begin{abstract}
The Northeast of Brazil is characterized by a semi-arid climate and irregular rainfall over time, which jeopardize agriculture production, public supply and other sectors. In this sense, research activities that promote Sustainable Development provide subsidies for the implementation of new public policies aimed at water sustainability in the region. The purpose of the present research was to compare rainfall data obtained from pluviometric stations of the Fundación Cearense de Metrología y Recursos Hídricos (FUNCEME) (translated as Cearense Foundation of Meteorology and Water Resources), with precipitation estimates obtained by means of the Tropical Rainfall Measuring Mission (TRMM) satellite, from January 1, 1998 to December 31, 2017, totaling twenty years of analysis for the entire state of Ceará, Brazil. In its first part, the research employed the techniques of comparing pixel to point (A and B), point to pixel (C), and pixel to pixel (D) along with inverse distance weighted (IDW) interpolation; in the second stage, data were analyzed by accuracy evaluation metrics: mean absolute error (MAE), root-mean-square error (RMSE) and the correlation coefficient (r). Analyzes results indicated TRMM satellite imagery to be a good alternative with $16.46 \mathrm{~mm}$ MAE, $26.78 \mathrm{~mm}$ RMSE and a correlation coefficient of 0.96 .
\end{abstract}

Keywords: Water Resources, Rainfall, Remote Sensing.

\title{
Resumen
}

El Nordeste de Brasil, se caracteriza por el clima semiárido y la irregularidad de las lluvias a lo largo del tiempo, que comprometen la agricultura, el abastecimiento público y otros sectores. En este sentido, investigaciones que promuevan el Desarrollo Sostenible dan subsidios para la implementación de nuevas políticas públicas dirigidas a la sostenibilidad hídrica de la región. Esta investigación tuvo por objetivo, comparar los datos de lluvia de las estaciones pluviométricas de la Fundación Cearense de Meteorología y Recursos Hídricos (FUNCEME), con las estimaciones de precipitación del satélite Tropical Rainfall Measuring Mission (TRMM), entre el 1 de enero de 1998 y 31 de diciembre de 2017, totalizando veinte años de análisis para todo el estado de Ceará, Brasil. En la primera parte la investigación utilizó las técnicas de comparación del píxel a punto (A y B), punto a píxel (C), y píxel a píxel (D), junto con la ayuda del interpolador Inverso del peso de la distancia (IDW), en la segunda etapa los datos fueron analizados por medidas métricas de exactitud: Error Medio Absoluto (EMA), Raíz del Error Medio cuadrático (REMQ) y el Coeficiente de correlación (r). Los resultados de los análisis, indicaron que la utilización de las imágenes del satélite TRMM se presentó como buena alternativa con el EMA de 16,46mm en la media, el REMQ de $26,78 \mathrm{~mm}$ y la correlación de 0,96 .

Palabras clave: Recursos Hídricos, Pluviometría, Percepción Remota. 


\section{Introdução}

O estudo do clima é de fundamental importância para entender dinâmica atmosférica terrestre, tendo em vista que auxilia no "planejamento estratégico dos recursos hídricos, subsidiando estudos associados ao balanço hídrico, controle de inundações, irrigação de culturas, modelagem hidrológica, monitoramento de seca e de geada e caracterização climatológica" (Louzada, 2016).

O ponto de partida para a construção desta pesquisa, inicia-se pela busca de compreender melhor o clima do estado do Ceará, especialmente quanto as chuvas. Para tanto, fez-se uso de imagens de satélite para quantificação das precipitações, procurando melhorar os dados de chuva justamente no momento em que a região passa por dos períodos de maior estiagem.

Diante disso, esta pesquisa se propôs a comparar e avaliar a qualidade das estimativas de chuva geradas a partir do satélite TRMM, produto 3B43_V7, na área que compreende o estado do Ceará num período correspondente a 20 anos, entre 1 de janeiro de 1998 a 31 de dezembro de 2017, aplicando diferentes métodos de comparação e aplicando testes estatísticos, considerando a grande quantidade de metodologias empregadas em outras pesquisas sobre a mesma temática.

O satélite TRMM (Tropical Rainfall Measuring Mission), lançado em 1997, permite obter dados de precipitação pluvial e resolução temporal em escala mensal e diária desde o ano 1998 (Pereira et al., 2013). O TRMM é formado por diversos produtos de estimativas de chuvas, cujo objetivo é o melhoramento da medição da precipitação tropical por sensoriamento remoto (Sodré et al., 2012; Aires et al., 2016).

Ao passarem pelos processos de comparação e avaliação, os dados de estimativas de precipitação derivada dos sensores remotos, podem se tornar informações complementares às coletadas em solo e até mesmo suprir a carência de dados de precipitação em determinadas regiões onde o monitoramento é precário ou inexistente (BERNARDI, 2016).

Assim, torna-se necessária uma avaliação dessas estimativas do TRMM no estado do Ceará, pois se os dados forem concordantes irá contribuir no entendimento da distribuição espacial e quantidade das chuvas. Com a evolução das tecnologias utilizadas para estimar a precipitação por sensoriamento remoto, aliado as técnicas de correção, torna-se cada vez 
mais viável a utilização dessas informações em colaboração mútua com as medições tradicionais em busca de melhores resultados.

\section{Área do Estudo}

A área de pesquisa corresponde ao Estado do Ceará, localizado entre as latitudes $02^{\circ} 47^{\prime} \mathrm{S}$ e $07^{\circ} 51^{\prime} \mathrm{S}$ e as longitudes $37^{\circ} 14^{\prime} \mathrm{O}$ e $41^{\circ} 24 \mathrm{O}$.

O estado do Ceará é pertencente a uma das 27 unidades federativas do Brasil, está situado na Região Nordeste limitando-se a norte com o Oceano Atlântico, ao sul com o Estado de Pernambuco; a leste com os Estados do Rio Grande do Norte e Paraíba e a oeste com o Estado do Piauí. A área total é de $148.926,472 \mathrm{~km}^{2}$ e a população encontra-se espalhada por 184 munícipios, sendo estimada em de 9.075.649 habitantes, (IBGE, 2018).

Figura 1. Mapa de Localização do Estado Ceará.

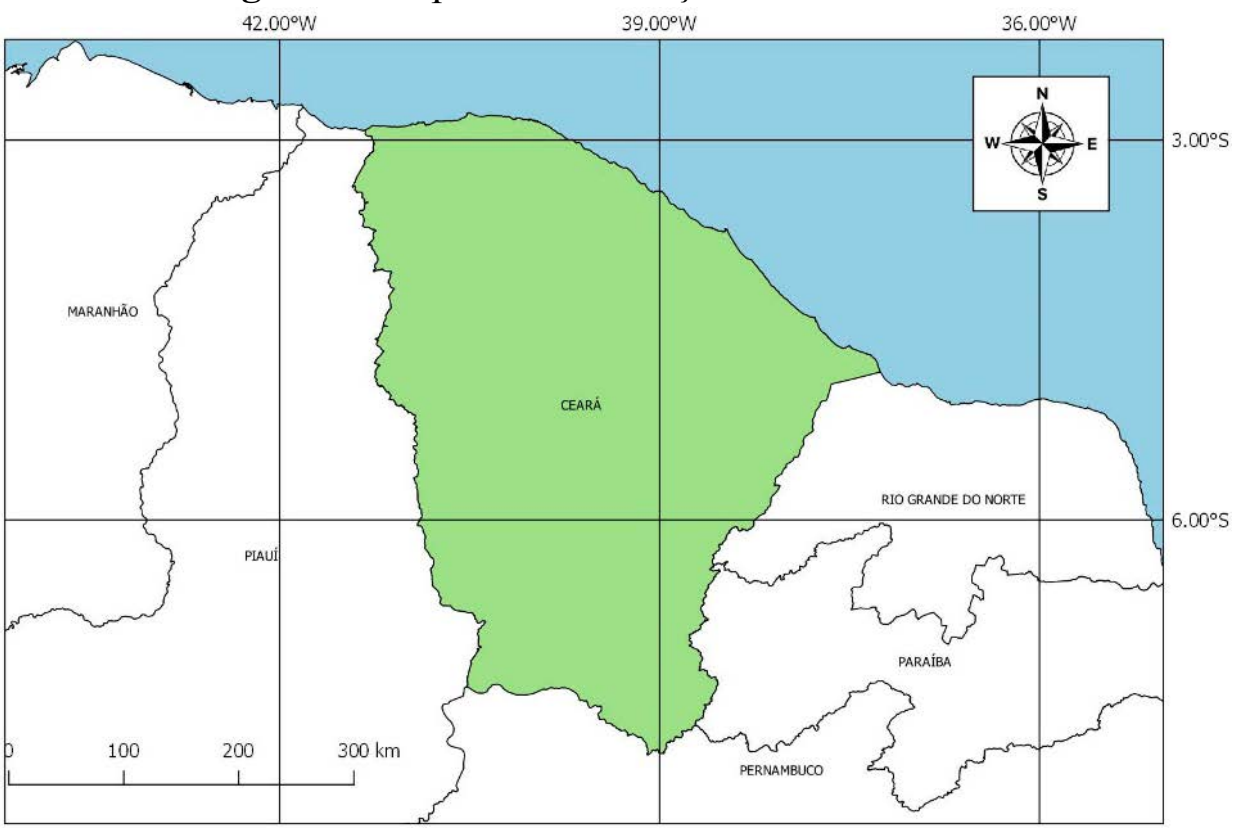

Fonte: MMA, adaptado pelo autor, 2018).

O Ceará é rodeado por formações de relevo relativamente altas de serras e chapadas, sendo a oeste delimitado pela Serra da Ibiapaba; a leste, parcialmente, pela Chapada do Apodi; ao sul, pela Chapada do Araripe; e 
na área central é composta pela Depressão Sertaneja e pelos maciços, com destaque para o Maciço do Baturité e Serra da Meruoca.

Segundo Brandão et al. (2014), a diversidade geológica do Ceará se distingue em dois domínios geológicos: rochas sedimentares e rochas cristalinas. As rochas sedimentares são encontradas nas chapadas e no litoral e são as mais importantes como aquífero com excelentes condições de armazenamento e fornecimento d'água. Já as rochas cristalinas são encontradas na Depressão Sertaneja, com permeabilidade muito baixa e tem baixo potencial hídrico, o que contribui para os efeitos do clima semiárido na região.

O clima do Ceará é predominantemente semiárido e tropical-seco, onde as regiões mais áridas, também chamada de Sertão Cearense, se localizam na Depressão Sertaneja, com pluviosidades que, podem ser menores que $500 \mathrm{~mm} / \mathrm{ano}$, mas também podem se aproximar de $1.000 \mathrm{~mm} /$ ano em outras áreas caracterizadas pelo clima semiárido brando, a exemplo de algumas áreas da Chapada do Araripe, Serra da Ibiapaba, Maciço do Baturité e na faixa litorânea (FUNCEME, 2014).

\section{Metodologia}

De modo geral, esta pesquisa consiste em uma análise comparativa entre as precipitações observadas em postos pluviométricos da FUNCEME no estado do Ceará, com as precipitações estimadas pelo satélite TRMM, através do produto 3B43_V7.

Os dados de precipitação que foram utilizados, são provenientes da rede de postos pluviométricos da FUNCEME, que disponibiliza os dados em seu site. Para esta pesquisa foi considerado o período de 20 anos, entre 1 de janeiro de 1998 a 31 de dezembro de 2017, de forma a abranger período comum ao início das estimativas do TRMM.

Enquanto os dados do TRMM foram utilizados os pixels provenientes do produto 3B43_V7, que corresponde as estimativas mensais de precipitação, com resolução espacial de $0,25^{\circ}$ de latitude e $0,25^{\circ}$ de longitude respectivamente, também no mesmo período dos 20 anos já citado.

Para análise, interpolação, comparação e espacialização dos dados de precipitação foi utilizado o software livre QGIS 2.18. Para aplicação dos testes estatísticos, foi utilizado o software livre de estatística Rstudio 1.0.153, uma interface gráfica ao usuário (em inglês Graphical User 
Interface - GUI) para o R, além do programa de planilha eletrônica Microsoft Excel 2013.

Os dados de precipitação foram obtidos a partir das séries históricas dos postos pluviométricos da FUNCEME que estão no formato txt. Já quanto a seleção dos postos, foi a partir de dois critérios, sendo o primeiro em relação ao tempo, tendo em vista que o TRMM começou a gerar seus dados partir de 1 de janeiro de 1998 e a segunda pelos postos que não apresentaram falhas e/ou descontinuidades nos dados, a fim de obter uma série histórica completa e apta ao desenvolvimento da pesquisa, a quantidade de postos está descrita na Tabela 1 .

Tabela 1. Seleção dos postos pluviométricos.

\begin{tabular}{|c|c|c|}
\hline Situação do Posto Pluviométrico & Quantidade & Total (\%) \\
\hline Total de Postos FUNCEME & 850 & $100 \%$ \\
Fora de Tempo do Período Estudado & 537 & $63,17 \%$ \\
Ausência de Dados & 80 & $9,41 \%$ \\
Fechados & 50 & $5,88 \%$ \\
Postos utilizados na pesquisa & $\mathbf{1 8 3}$ & $\mathbf{2 1 , 5 2 \%}$ \\
\hline
\end{tabular}

Do total de 850 postos, restaram 183 que atenderam aos requisitos da pesquisa. Estes postos pluviométricos encontram-se espalhados ao longo do estado do Ceará e fornecem dados diários de precipitação, para combinar com o TRMM que estima precipitação mensal, os dados da FUNCEME foram organizados em acumulados mensais também. 
Figura 2. Mapa dos postos pluviométricos utilizados na pesquisa.
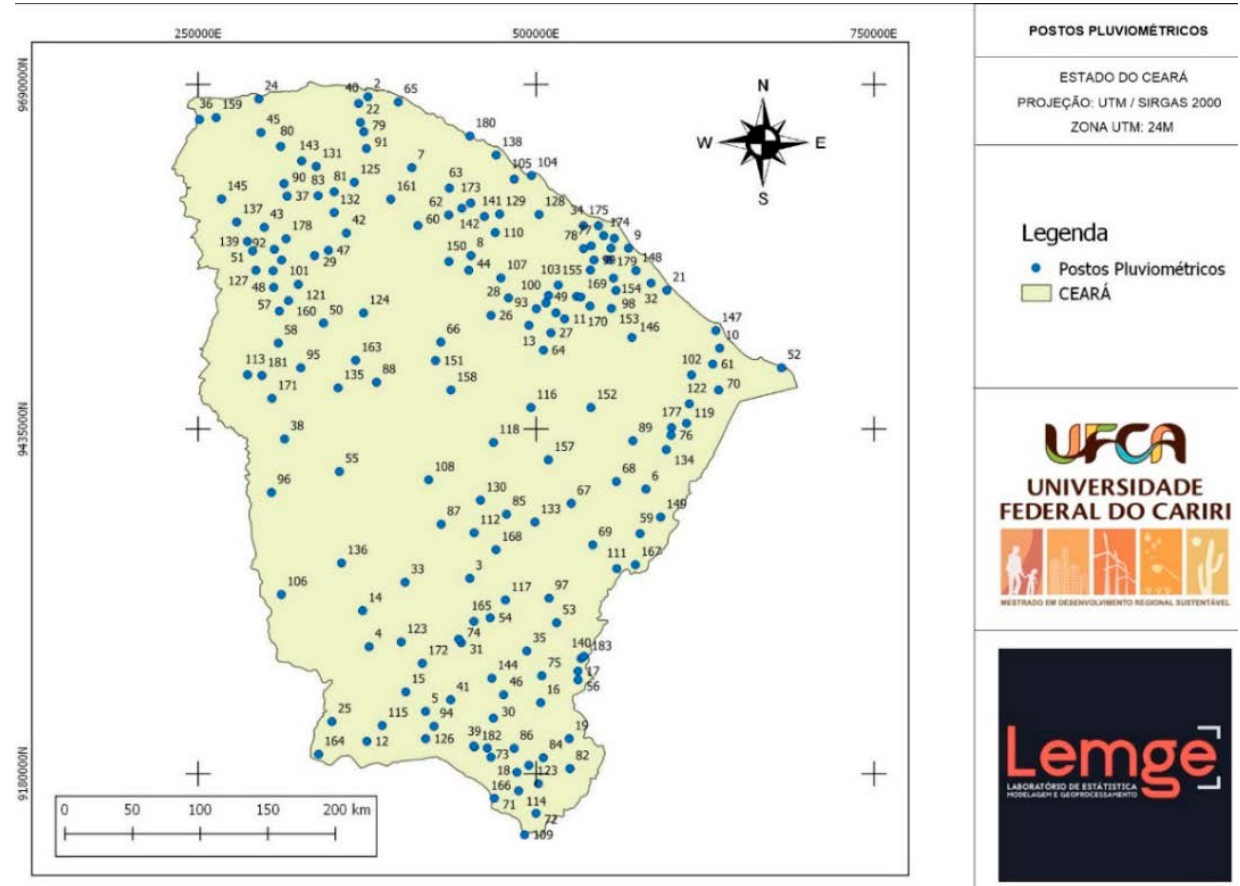

Fonte: autor.

Os dados do TRMM são disponibilizados através do produto 3B43 V7, que corresponde aos valores mensais de chuva e estão disponíveis nos formatos GeoTIFF e kml no site GIOVANNI da NASA. Para esta pesquisa foram utilizados dados no formato GeoTIFF, que correspondem a pixels na resolução espacial de $25 \mathrm{~km}$ por $25 \mathrm{~km}$, conforme Figura 3. 
Figura 3. Mosaico de pixels do TRMM com valores mensais de precipitação
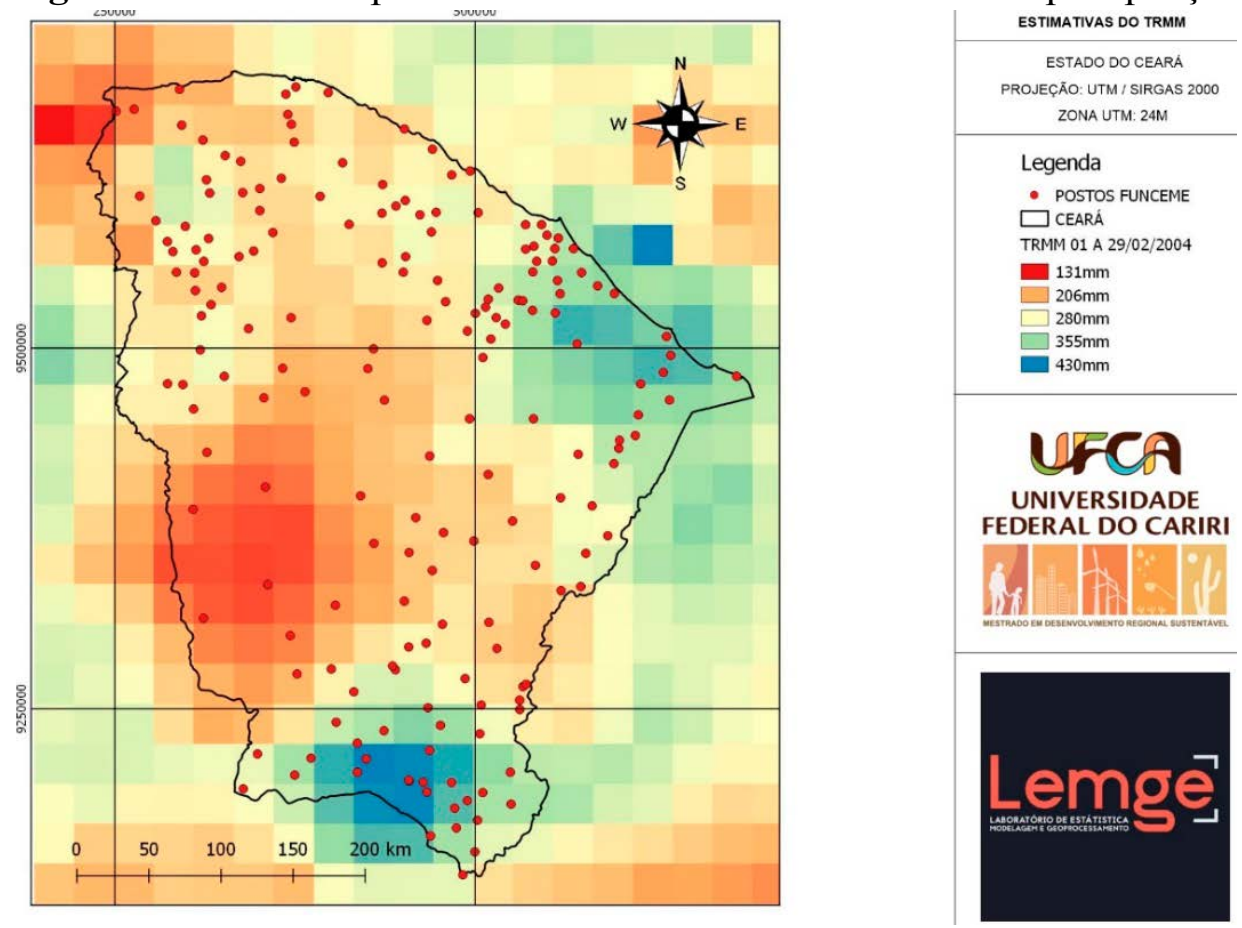

Fonte: autor, 2019.

\section{Metodologias de Comparação}

A precipitação em si é uma informação pontual e localizada, enquanto o TRMM mede a chuva média num determinado pixel $(25 \times 25 \mathrm{~km})$, por sua vez, o posto pluviométrico mensura um dado localizado, podendo haver diferenças consideráveis de um posto próximo a outro. Diante dessa situação, a literatura especializada tem aplicado diversas metodologias para comparar dados obtidos por sensoriamento remoto e dados terrestres, não existindo um modelo padronizado de verificação dos dados estimados por satélite.

Por tanto, para efeito desta pesquisa, foram adotados 4 métodos de comparação (Quadro 2) entre os dados estimados e observados, e que se basearam em outros trabalhos nessa área de pesquisa, sendo: 
- Comparação A (pixel a ponto): os dados estações pluviométricas, foram interpolados no método Inverso do Peso da Distância (inglês Inverse Distance Weighted - IDW) e comparados com a série do TRMM cujo centro de pixel está mais próximo do pluviômetro. Essa maneira de comparar as estimativas do satélite é vantajosa pois não altera nenhum dos componentes da comparação do dado monitorado e a estimativa. Exemplos de trabalhos que utilizaram essa mesma metodologia: Liu e Peter (2013) em bacias hidrográficas do Taiwan; Almeida et al. (2015), no estado do Amazonas; Bernardi (2016), no estado do Rio Grande do Sul e Soares (2016) para o estado da Paraíba.

- Comparação B (pixel a ponto): essa comparação segue o mesmo modelo realizado na A, sendo que neste caso quem foi interpolado (IDW) foram os dados do TRMM. Dessa maneira, as estimativas do satélite foram comparadas com as séries das estações pluviométricas, conforme Chen et al., (2013).

- Comparação C (ponto a pixel): neste caso os dados foram comparados sem passar por nenhum tipo de processamento, diretamente como são baixados, conforme trabalhos de Massagli et al., (2011), para a bacia hidrográfica do Rio Ji-Paraná - Rondônia; Pombo e Oliveira (2015) em Angola; Silva et al., (2016) para a Região do Alto Rio Paraíba e Soares (2016) no estado da Paraíba.

- Comparação D (pixel a pixel): nesta comparação, os dados oriundos dos pluviômetros foram interpolados (IDW) para uma grade de mesmas dimensões das estimativas do TRMM, de modo que a sobreposição do pixel e a grade interpolada tivessem o mesmo tamanho, como mostrado nos trabalhos de Pereira et al. (2013); Morais e Ferreira (2015) no estado do Goiás e Melo et al. (2015) em todo Brasil.

Também foi utilizado a interpolação Inverso do Peso da Distância (no inglês Inverse Weight of Distance - IDW), pois devido ao número reduzido e muitas vezes insuficientes de dados na variabilidade espacial, a ferramenta acaba sendo ideal para dispersão dos valores localizados e fornecer uma boa representatividade espacial aos eventos de chuva, (GREGO et al., 2017). Para isso, utilizou uma adaptação dos métodos utilizados por Silva et al. (2016) e Soares (2016) efetuando uma combinação entre 
estimativas de satélite com medições reais, através do interpolador Inverso do Peso da Distância.

Além das comparações descritas acima, foram utilizadas 2 medidas que quantificam o erro associado às estimativas de chuva. A primeira foi o Erro Médio Absoluto (EMA), Equação 1, que corresponde à média das diferenças absolutas entre os valores observados e estimados:

$$
E M A=\frac{\sum_{i=1}^{n}|E i-O i|}{n}
$$

E a segunda foi a Raiz do Erro Médio Quadrático (REMQ), Equação 2 , que é a raiz quadrada da média do quadrado das diferenças entre os dados de precipitação estimadas pelo satélite e as obtidas pelos pluviômetros:

$$
R E M Q=\sqrt{\frac{\sum_{i=1}^{n}(E i-O i)^{2}}{n}}
$$

O percentual do Bias que em português que dizer viés, foi utilizado para medir a tendência média dos dados simulados (maiores ou menores que os valores observados), e pode ser calculado através da Equação 3:

$$
\text { Pbias }=\sum_{i=1}^{n} \frac{(E i-O i)}{\sum_{i=1}^{n} O i} \cdot 100
$$

Além disso, foi estimado o coeficiente de correlação (r) equação 4, que mede o grau da correlação entre os valores das estimativas de chuva do satélite com os observados. O coeficiente de correlação, reflete a extensão de uma relação linear entre dois conjuntos de dados, que varia entre -1, 0 e 1. Para isto será necessário aplicar o método através da Equação 4:

$$
r=\frac{(E-\bar{E}) \cdot(O-\bar{O})}{\sqrt{\sum_{i=1}^{n}(E i-\bar{E})^{2} \cdot \sum_{i=1}^{n}(O i-\bar{O})^{2}}}
$$


Onde:

$E_{i}:$ corresponde o valor estimado pelo satélite no intervalo de tempo $i$;

$O i$ : é valor observado em superfície no intervalo de tempo $i$;

$n$ : é número de dados analisados;

$\bar{E}$ : é valor médio estimado pelo satélite e por fim,

$\bar{O}:$ é valor médio observado em superfície.

Figura 4. Diagrama dos métodos Comparativos

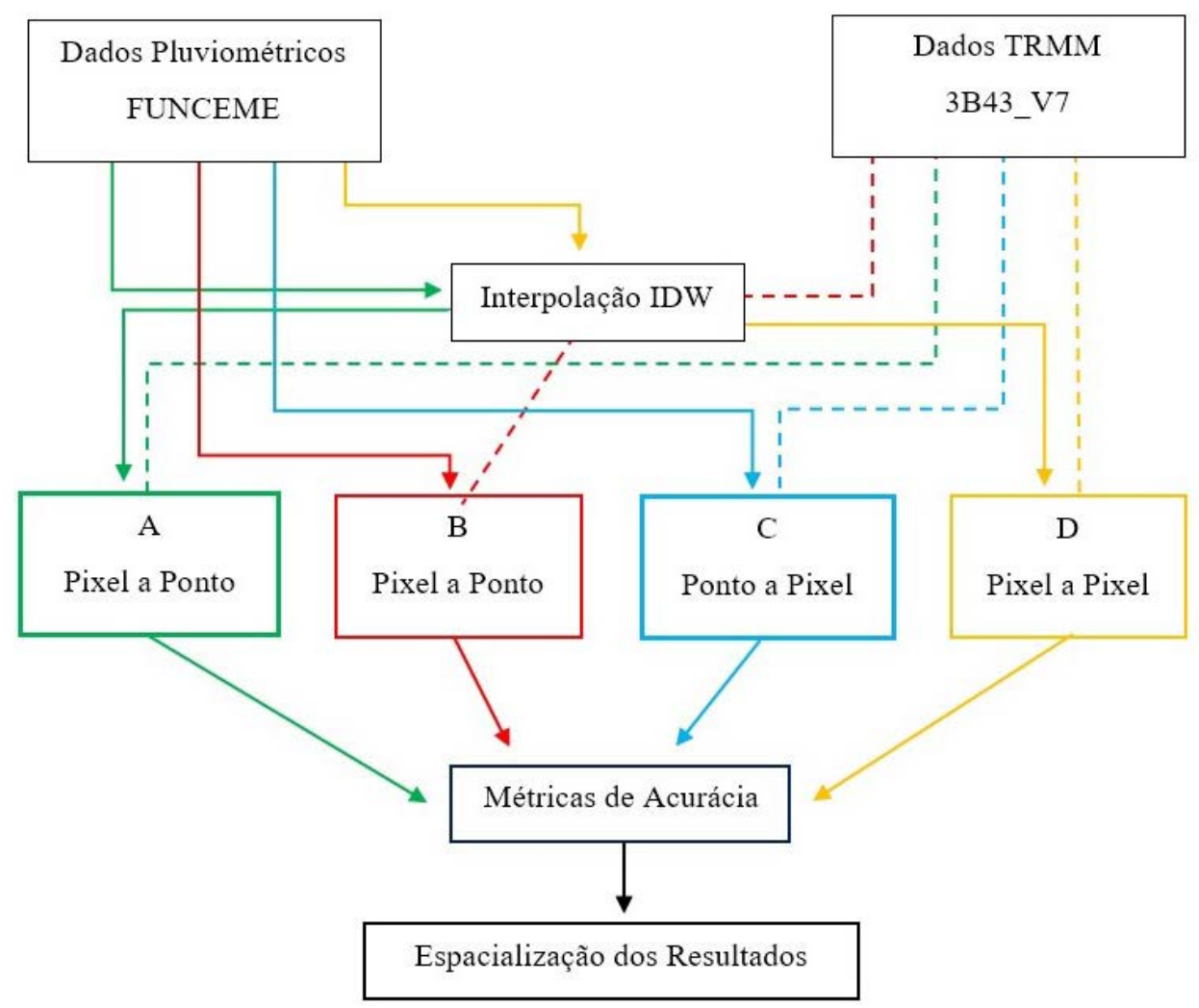

Fonte: autor, 2019.

\section{Resultados e Discussões}

Para obtenção dos resultados, cada conjunto de dados das comparações A, B, C e D que podem ser representados tanto por pontos ou pixels, foram submetidos as métricas de comparação das equações 1, 2, 3 e 4, referente para os 20 anos da série histórica analisada nesta pesquisa. 


\section{- $\quad$ Erro Médio Absoluto - EMA}

O EMA, representa a média das diferenças entre os totais de precipitação estimada e da observada nos postos pluviométricos para o período de 1998 a 2017 em mm, sendo que, quanto menor for o erro, melhor a confiabilidade do dado estimando pelo TRMM.

$\mathrm{Na}$ Figura 5 tem-se a média das diferenças entre os totais de precipitação estimada e da observada nos postos pluviométricos do Estado do Ceará para o período de 1998 a 2017.

Figura 5. Mapa dos valores médios do EMA para as

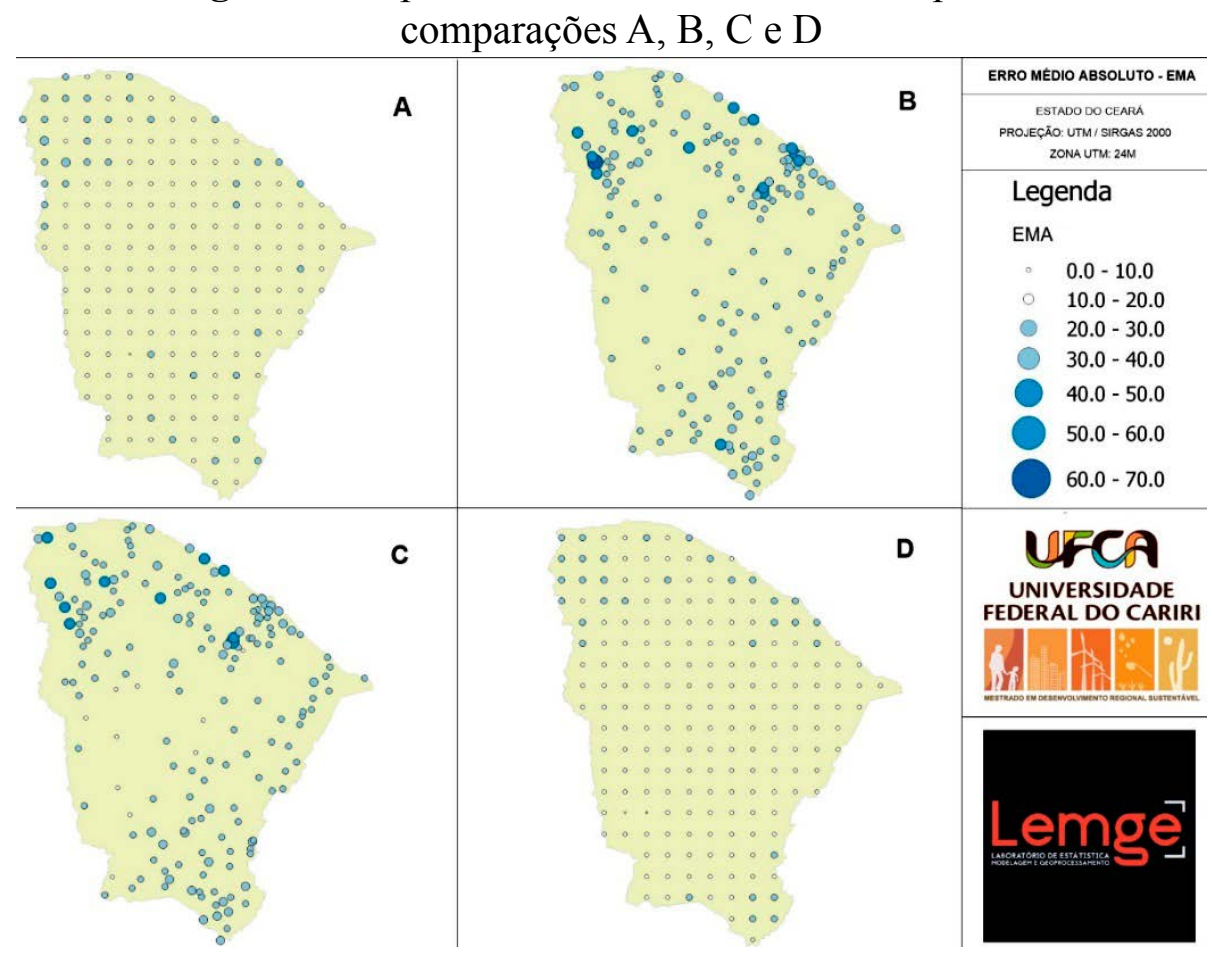

Fonte: autor, 2019.

De acordo com a Figura 5, observa-se que os menores valores médios do EMA ocorreram nas comparações D e A, tendo médias de $16,46 \mathrm{~mm}$ e $16,88 \mathrm{~mm}$ respectivamente, como pode ser verificado na Tabela 2. 
Tabela 2. Demonstra valores obtidos com EMA.

\begin{tabular}{|c|c|c|c|c|}
\hline EMA & Mínima & Máxima & Média & CV (\%) \\
\hline A & 9,76 & 34,74 & 16,88 & 25,96 \\
B & 18,20 & 65,69 & 29,10 & 22,40 \\
C & 14,05 & 61,78 & 28,17 & 23,12 \\
D & $\mathbf{9 , 3 2}$ & $\mathbf{2 9 , 2 8}$ & $\mathbf{1 6 , 2 7}$ & $\mathbf{2 0 , 7 1}$ \\
\hline
\end{tabular}

Verifica-se que a comparação D apresentou o menor erro tanto para os valores máximos e mínimos. Já as comparações $\mathrm{B}$ e $\mathrm{C}$ apresentaram maiores erros, chegando a ter 65,69 e $61,78 \mathrm{~mm}$ respectivamente. Por tanto, o EMA variou entre 9,32 a 65,69 mm, sendo que as comparações A e D obtiveram resultados praticamente iguais, tendo uma diferença na média do EMA de apenas 2,5\% entre elas.

No Gráfico 1, tem-se as comparações dos valores obtidos do TRMM e dos postos pluviométricos do Estado do Ceará. Nota-se que, apesar do Coeficiente de Variação (CV) ter demonstrado resultados para as comparações entre D e A praticamente iguais para os 20 anos de dados analisados, os valores em A apresentaram dados mais discrepantes se comparado a D, chegando a $25,96 \%$, enquanto D variou $20,57 \%$. Já as comparações B e C obtiveram comportamento mais semelhantes.

Gráfico 1. Gráfico de box-plot do EMA com as comparações entre TRMM e postos pluviométricos, para todo estado do Ceará de 1998 a 2017.

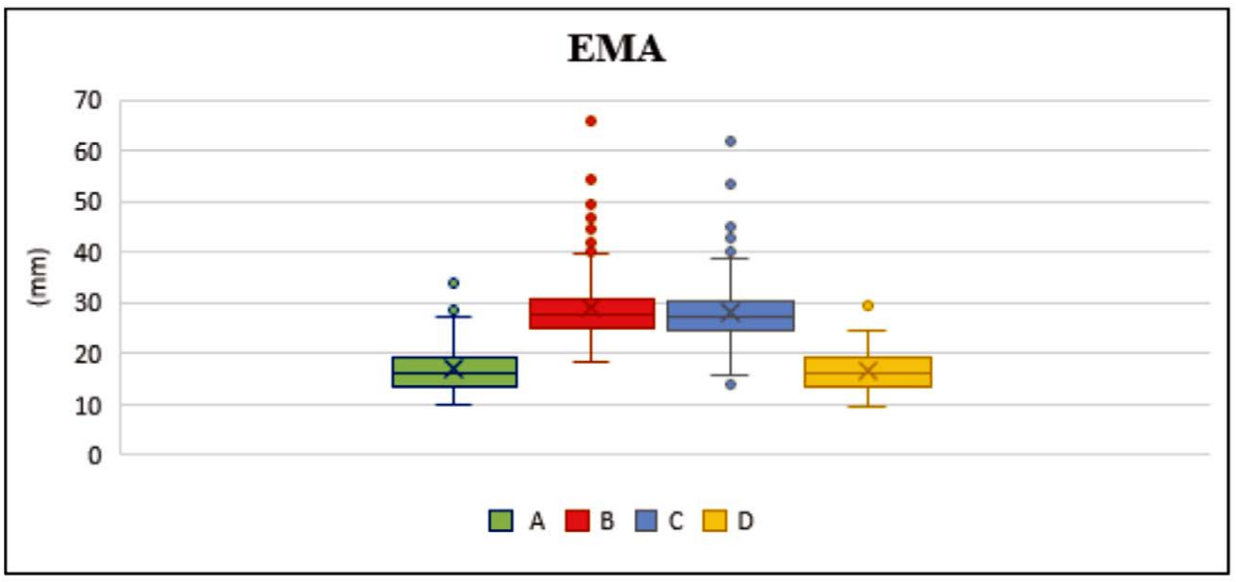




\section{- Raiz do Erro Médio Quadrático - REMQ}

A REMQ, do mesmo modo que o EMA, calcula a média dos quadrados dos desvios entre os valores observados e estimados, sendo compassivo a erros com valores absolutos maiores. No entanto, fornece maiores ponderações aos pesos quanto aos erros espaciais quando comparado ao EMA.

$\mathrm{Na}$ Figura 6 pode-se verificar os resultados das comparações analisadas a partir do REMQ, correspondendo a acurácia das estimativas em $\mathrm{mm}$, sendo relevante para analisar o grau de confiabilidade das estimativas. Nela verifica-se que as médias com maiores valores de erros estão localizados na porção norte ocidental do estado, no entorno da Serra Grande e na Região Metropolitana de Fortaleza, chegando a quase $110 \mathrm{~mm}$ de erro no caso da comparação B.

Figura 6. Mapa dos valores médios do REMQ para as comparações A,

$$
\mathrm{B}, \mathrm{C} \text { e D }
$$

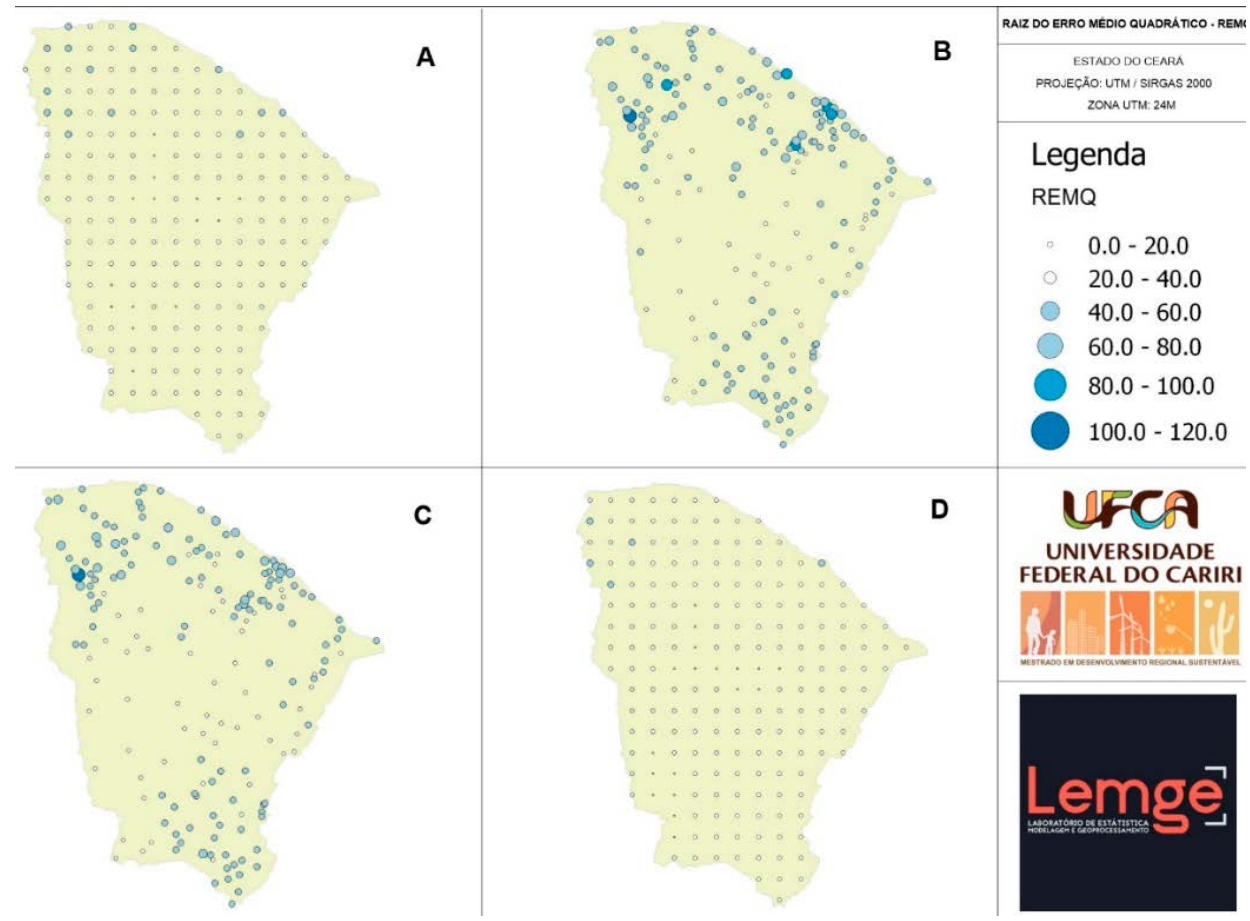

Fonte: autor, 2019. 
A Tabela 3 demonstra que a REMQ variou entre 15,26 a 109,65 mm. Novamente a comparação D obteve o melhor desempenho, novamente tanto a comparação D como A tiveram resultados aproximados com 2,37\% de diferença entre elas.

Tabela 3. Demonstra valores obtidos com REMQ.

\begin{tabular}{|c|c|c|c|c|}
\hline REMQ & Mínima & Máxima & Média & CV (\%) \\
\hline A & 15,70 & 58,19 & 27,52 & 27,46 \\
B & 29,71 & 109,65 & 48,14 & 25,96 \\
C & 24,35 & 102,17 & 46,07 & 24,05 \\
D & $\mathbf{1 5 , 2 6}$ & $\mathbf{4 8 , 4 4}$ & $\mathbf{2 6 , 4 3} \mathbf{C}$ & $\mathbf{2 1 , 8 6}$ \\
\hline
\end{tabular}

No Gráfico 2, pode-se visualizar melhor os resultados da REMQ, para todas as comparações, onde a linha central dentro de cada box-plot representa a média de cada comparação analisada no estudo. Nota-se que o Coeficiente de Variação (CV) foi menor na comparação D de 21,86\%, enquanto as comparações $\mathrm{A}, \mathrm{B}$ e $\mathrm{C}$ apresentaram dados mais discrepantes se comparado a D.

Gráfico 2. Gráfico de box-plot da REMQ com as comparações entre TRMM e postos pluviométricos, para todo estado do Ceara de 1998 a 2017.

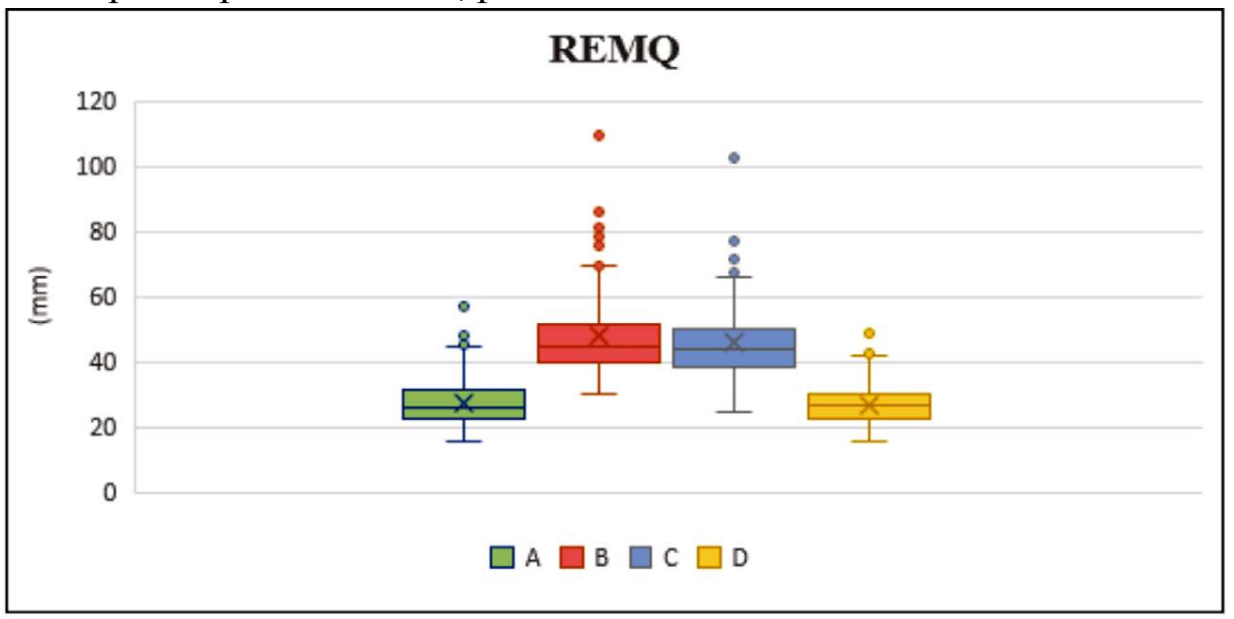


Em suma as duas métricas (EMA e a REMQ) apresentaram comportamentos parecidos na variabilidade dos erros encontrados, onde os menores valores correspondem a menor diferença entre as precipitações estimadas pelo TRMM.

\section{- Pbias}

O percentual de viés (PBIAS) mede a tendência de os dados estimados serem maiores ou menores que os observados. Valores positivos indicam viés de superestimação, enquanto valores negativos indicam viés de subestimação do modelo. Se o Pbias for igual a zero, significa que o valor estimado é igual ao valor observado.

Olhando a Figura 7, percebe-se que houve uma tendência de subestimação do TRMM na distribuição do Pbias para as comparações B e C, tanto na porção norte ocidental do estado, no entorno da Serra Grande, quanto no entorno da região metropolitana de Fortaleza.

Figura 7. Mapa dos valores do Pbias para as comparações A, B, C e D

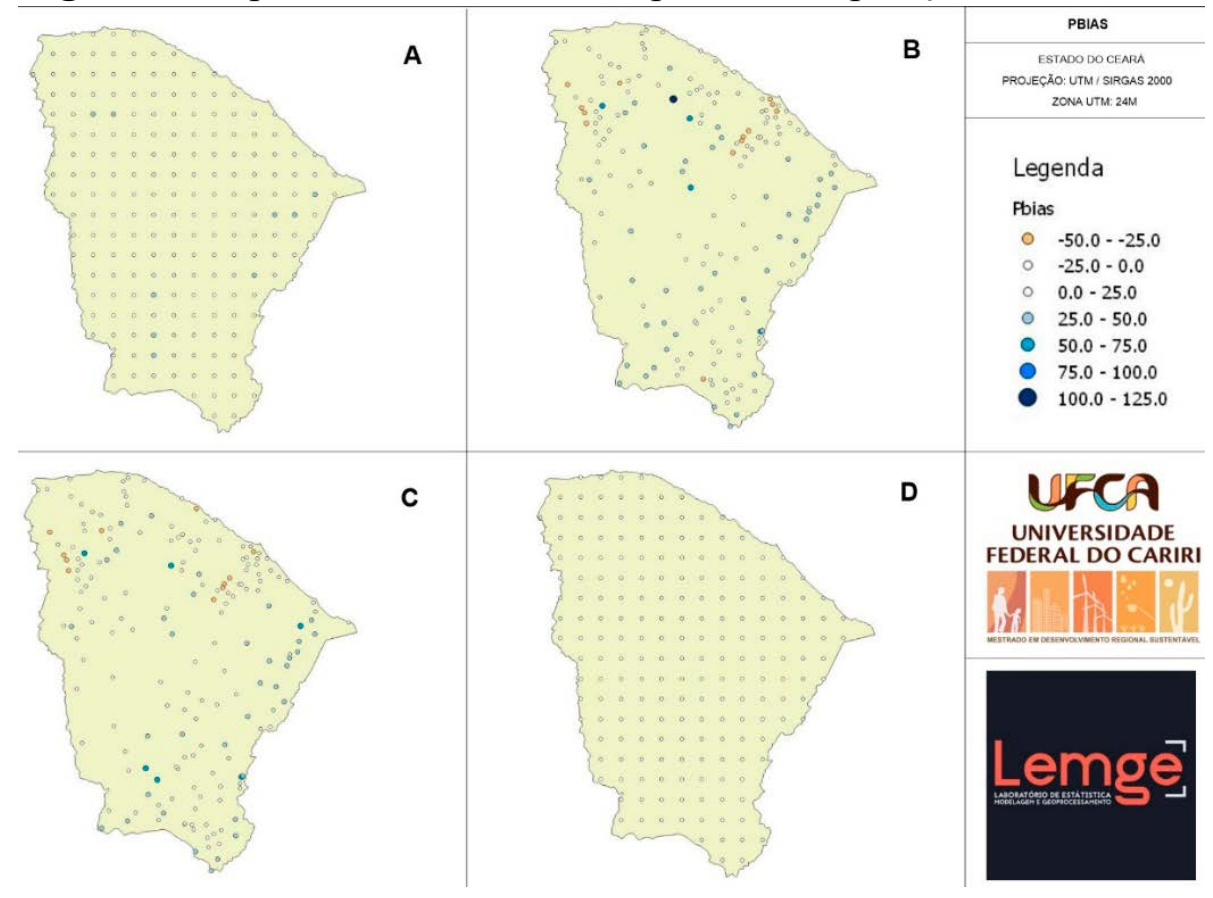

Fonte: autor, 2019. 
Na média para todos os postos, o valor do TRMM, foi positivo, indicando assim uma leve superestimativa dos valores do TRMM em relação aos dados de pluviometria coletados nas estações pluviométricas (Tabela 4).

Tabela 4. Valores obtidos com PBIAS para os quatros métodos de comparação.

\begin{tabular}{|c|c|c|c|c|}
\hline PBIAS & Mínima & Máxima & Média* & CV (\%) \\
\hline A & $-24,32$ & 34,15 & 4,21 & 299,69 \\
B & $-43,56$ & 118,48 & 8,93 & 260,86 \\
C & $-41,15$ & 125,98 & 10,76 & $\mathbf{2 1 0 , 1 0}$ \\
D & $\mathbf{- 2 4 , 0 4}$ & $\mathbf{2 3 , 1 9}$ & $\mathbf{3 , 1 5}$ & 354,87 \\
\hline
\end{tabular}

Novamente no Pbias, a comparação D obteve o menor valor médio de 3,15 e a comparação A também obteve um valor aproximado, de 4,21 demonstrando uma tendência mais aproximadas do 0 (zero). Já as comparações B e C tiveram uma variação grande entre os valores negativos e positivos, transportando assim, uma tendência maior de superestimar a precipitação estimadas, conforme demonstrado no Gráfico 3.

O Coeficiente de Variação (CV) apresentado na Tabela 7, demonstrou que a comparação $\mathrm{C}$ teve menor variância entre os dados de precipitação estimada e observada para os 20 anos, de 210,10\%, mesmo tendo a maior média dentre todas as comparações. 
Gráfico 3. Gráfico de box-plot do Pbias com as comparações entre TRMM e postos pluviométricos, para todo estado do Ceara de 1998 a 2017.

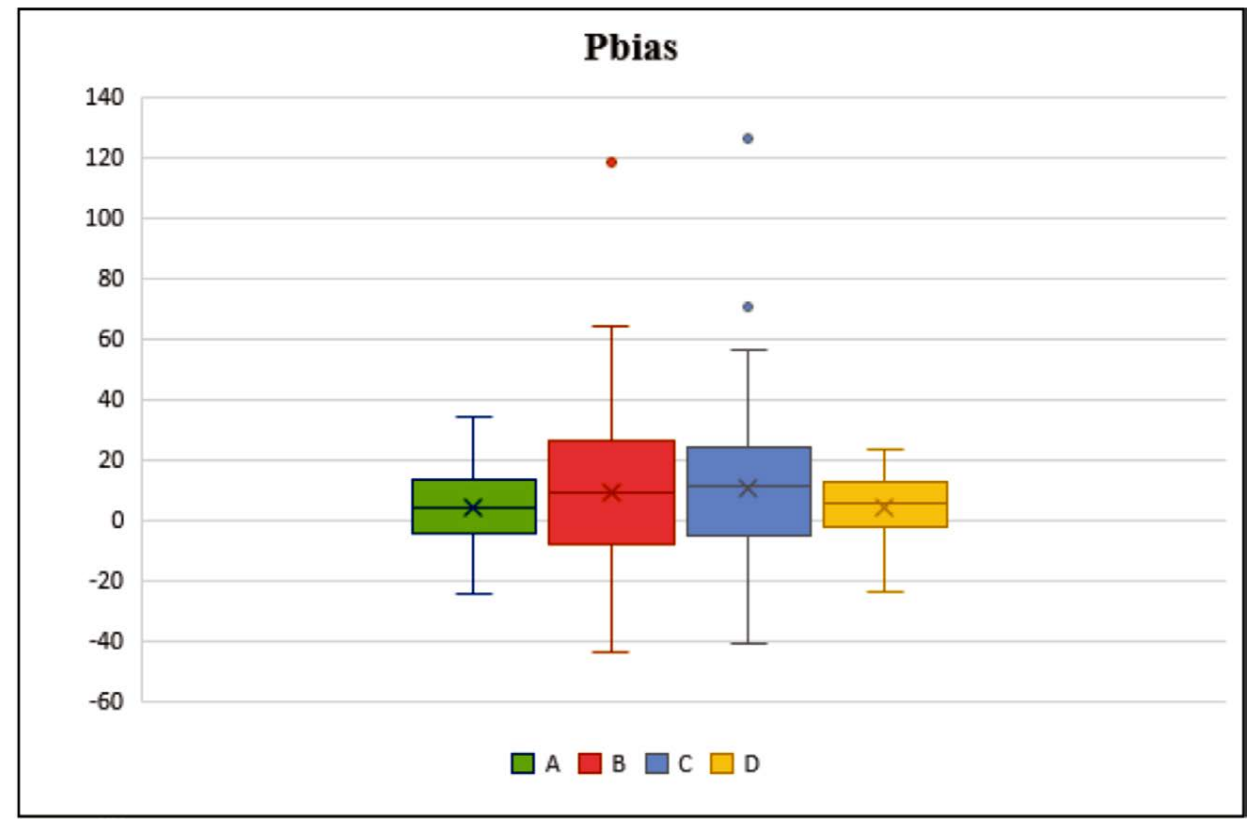

\section{Correlação (r)}

Para o valor de correlação de Pearson foi considerado pequeno (até $0,25)$, baixo $(0,26-0,49)$, moderado/médio $(0,50-0,69)$, alto/boa $(0,70$ 0,89 ) e muito alta/ótima (de 0,90 acima), de acordo com os valores de referência descritos por Louzada (2016).

O coeficiente de correlação variou entre 0,89 a 0,96 entre as médias, e novamente o resultado da comparação $\mathrm{D}$, se mostrou com maior a correlação entre a precipitação estimada pelo TRMM e a observada nos postos pluviométricos, apresentando assim melhor média para os valores acumulados. Na Figura 8, tem-se os mapas com a correlação entre pluviometria observada e estimada para o Estado do Ceará utilizando os quatro métodos de comparação (Quadro 1). 
Figura 8. Correlação entre pluviometria observada e estimada para as comparações A, B, C e D

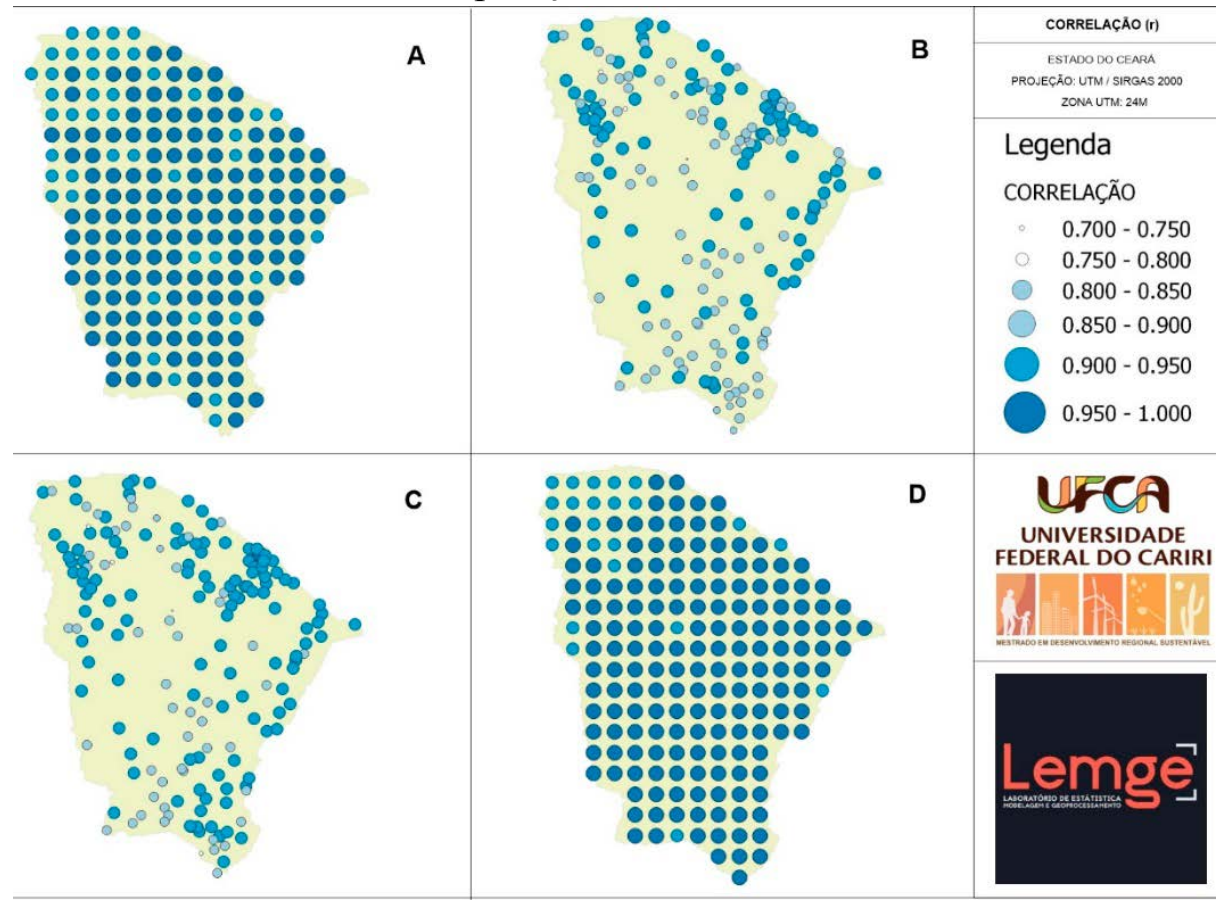

Fonte: autor, 2019.

O coeficiente de correlação variou de 0,89 a 0,96 entre as comparações analisadas, o que pode ser considerado um resultado muito satisfatório. Pode-se notar que as maiores correlações foram obtidas entre as comparações $\mathrm{D}$, A e C encontrando-se numa classificação de muito alta a ótima e a comparação B classificada como alta ou boa (Tabela 5). Neste cenário pode-se dizer que tanto a comparação $\mathrm{D}$ como A tiveram correlações praticamente iguais, com uma diferença de $1,05 \%$.

Tabela 5. Demonstra valores da correlação (r).

\begin{tabular}{|c|c|c|c|c|}
\hline r & Mínima & Máxima & Média* $^{*}$ & CV (\%) \\
\hline A & 0,90 & $\mathbf{0 , 9 7}$ & $0,95 \mathrm{~A}$ & 1,55 \\
B & 0,72 & 0,93 & $0,89 \mathrm{C}$ & 3,09 \\
C & 0,73 & 0,95 & $0,90 \mathrm{~B}$ & 3,18 \\
D & $\mathbf{0 , 9 3}$ & $\mathbf{0 , 9 7}$ & $\mathbf{0 , 9 6} \mathbf{A}$ & $\mathbf{0 , 9 9}$ \\
\hline
\end{tabular}


No Gráfico 4, observa-se que todas as comparações se aproximam de 1,0 , sendo que as comparações $\mathrm{D}$ e A tem menores variações nos dados comparados. Na Tabela 8 nota-se que, apesar do Coeficiente de Variação (CV) ter demonstrado a comparação D obteve menor variação de $0,93 \%$ e A foi a comparação obteve resultado também satisfatório de $1,55 \%$ para os 20 anos de dados analisados, já os valores de $\mathrm{B}$ e $\mathrm{C}$ apresentaram dados mais discrepantes se comparado a $\mathrm{D}$, chegando a $3,09 \% \mathrm{em} \mathrm{B}$, enquanto $\mathrm{C}$ variou $3,18 \%$. As comparações B e C obtiveram comportamento semelhante.

Gráfico 4. Gráfico de box-plot da correlação (r) das comparações entre TRMM e postos pluviométricos, para todo estado do Ceara de 1998 a 2017.

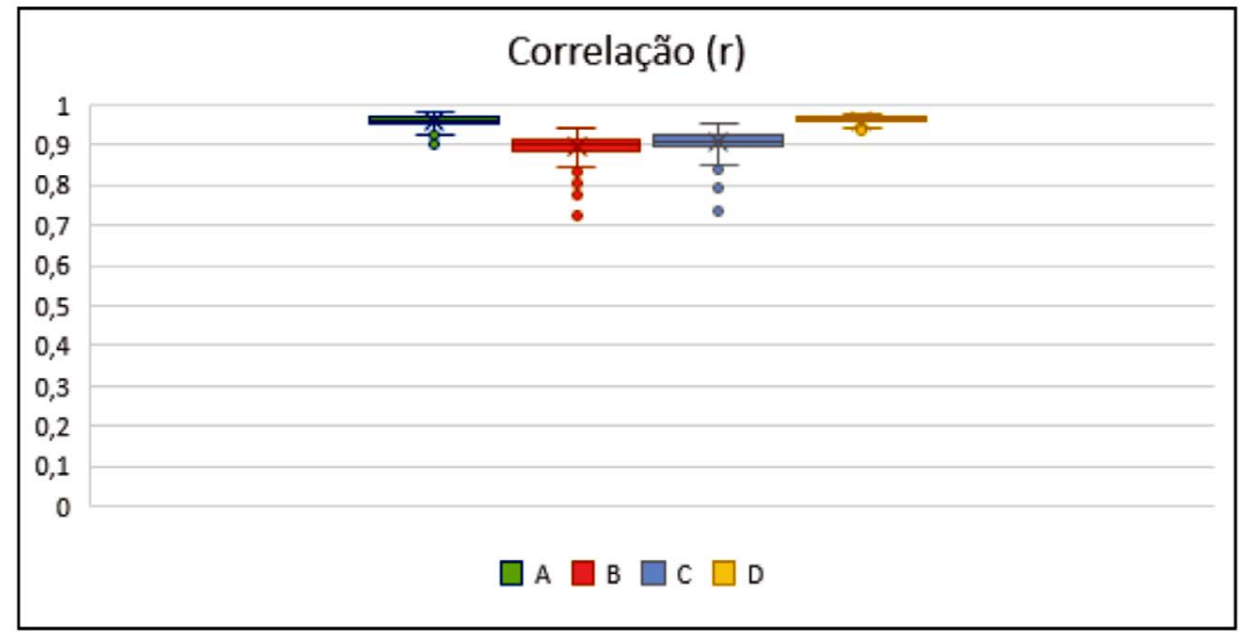

\section{Conclusão e Considerações Finais}

De modo geral, os valores estimados com o satélite TRMM foram aproximados com os dados observados em solo pelos postos pluviométricos. Sendo a comparação D a que obteve menor erro nas métricas de acurácia, menor percentual de erro no Pbias e a maior correlação entre todas as comparações analisadas nesta pesquisa, porém vale ressaltar que a comparação A obteve praticamente o mesmo desempenho, sendo também um modelo de comparação satisfatório (Tabela 6).

O índice de Pbias apresentou valores positivos, para todas comparações, o que indica que a utilização dos dados do TRMM tende a superestimar os valores observados de superfície. 
Tabela 6. Resumo dos resultados para todas as comparações.

\begin{tabular}{|c|c|c|c|c|}
\hline & A & B & C & D \\
\hline EMA & 16,88 & 29,10 & 28,17 & $\mathbf{1 6 , 2 7}$ \\
REMQ & 27,52 & 48,14 & 46,07 & $\mathbf{2 6 , 4 3}$ \\
PBIAS & 4,21 & 8,93 & 10,76 & $\mathbf{3 , 1 5}$ \\
r & 0,95 & 0,89 & 0,90 & $\mathbf{0 , 9 6}$ \\
\hline
\end{tabular}

A confiabilidade do TRMM em representar estimativas de chuvas para o estado do Ceará, comprova a eficácia de seus instrumentos em fornecer dados para o monitoramento climático a que se propôs. Lembrando que estes dados são disponibilizados para consulta a qualquer um que pesquise sobre a área e em qualquer lugar da Terra, possibilitando pesquisas em vários segmentos de acordo com necessidades de cada pesquisa.

Verificou-se nas comparações pelos quatro métodos, que os locais que apresentam os maiores erros, são justamente onde tem a maior concentração de postos pluviométricos para o mesmo pixel, significando que quanto maior o número de dados disponíveis em um mesmo pixel, maior é a tendência desses valores se diferenciarem da precipitação estimada pelo TRMM, mas que pode ser corrigido com a utilização da interpolação para ajuste espacial desses dados.

Pode-se considerar que a quantidade de pesquisas envolvendo os produtos do TRMM mostrou-se viável, mas apenas para alguns estudos específicos, considerando que os dados se comportam de maneira diferente para cada região do planeta. Como no caso do nordeste do Brasil, que possui clima semiárido, onde a radiação solar é alta e a cobertura de nuvem é baixa durante a maior parte do ano, associado a um menor índice de pluviosidade em relação a outras regiões do país, acaba favorecendo um bom desempenho dos sensores do TRMM.

Contudo, nota-se que há uma grande quantidade de métodos utilizados para avaliação da qualidade das estimativas, alguns com foco em análises pontuais, outros em comparações espaciais, alguns fazendo uso de interpoladores e outros mantendo os dados intactos. Logo, deve-se ter cuidado ao definir o método dependendo do objetivo da análise. 
Jose Reginaldo Medeiros-Feitosa - Carlos Wagner Oliveira Comparative study of precipitation data from the TRMM satellite and pluviometric stations in the state of Ceará, Brazil

\section{Referências}

Aires, U. R. V., Neto, J. de O. M. \& Mello, C. R. (2016). Estimativas de precipitação derivadas do satélite TRMM para a bacia hidrográfica do rio Paraopeba, MG, Revista Scientia Agraria - SA, 17(2), 57-66. Curitiba - PR, abril/maio, 2016, Versão On-line ISSN 1983-2443.

Almeida, C. T., Delgado, R. C., Junior, J. F. O.,Gois, G. \& Cavalcanti, A. S. (2015). Avaliação das estimativas de precipitação do produto 3B43TRMM do Estado do Amazonas. Floresta e Ambiente, 279-286.

Bernardi, E. C. S. (2016). Qualidade das Estimativas de Precipitação do Satélite TRMM no Estado do Rio Grande do Sul. 2016. 166 f. Dissertação (Mestrado em Engenharias) - Universidade Federal de Santa Maria, Santa Maria.

Brandão, R. L.; Freitas, L. C. B. (2014). Geodiversidade do estado do Ceará / Organização - Fortaleza - CE, CPRM, 214 p, ISBN 978-85-7499-140-5.

Chen, S.; Hong, Y.; Gourley, J. J.; Huffman, G. J.; Tian, Y.; Cao, Q.; Yong, B.; Kirstetter, P.; Hu, J.; Hardy, J.; Li, Z.; Khan, S. \& Xue, Xianwu. (2013). Evaluation of the successive V6 and V7 TRMM multisatellite precipitation analisys over the Continental United States. Water Resources Research, 49, 8174-8186.

FUNCEME - Fundação Cearense de Meteorologia, série histórica de chuvas do Ceará, link $<$ http://www.funceme.br/produtos/script/chuvas/ Download_de_series_historicas.php>, acessado em 05/03/2018.

Grego, C. R.; Texeira, A. H. de C.; Leivas, J. F.; Bayma-Silva, G.; Manjolin, R. C. (2017). Interpolação de dados agrometeorológicos em duas densidades amostrais no norte de Minas Gerais. Revista Agrometeoros, Passo Fundo, 25(1), 1-89, ago 2017, ISSN 2526-7043.

Louzada, F. L. R. O. (2016). Balanço hídrico climatológico com dados estimados pelo satélite Tropical Rainfall Measuring Mission para a bacia hidrográfica do Rio Doce. Tese (Doutorado em Recursos hídricos e Geoprocessamento em sistemas agrícolas), Universidade Federal do Espírito Santo, Alegre - ES, 2016.

Liu, J. K. \& Peter, T. Y. (2013). Topographic correction of wind-driven rainfall for landslide analysis in Central Taiwan with validation from aerial and satellite optical images. Remote Sens., 5( 6), 2571-2589. 
Massagli, G. O., Victoria, D. C. \& Andrade, R. G. (2011). Comparação entre precipitação medida em estações pluviométricas e estimada pelo satélite TRMM, $5^{\circ}$ Congresso Interinstitucional de Iniciação Científica - CIIC 20119 a 11 de agosto de 2011 - Campinas, SP.

Melo, D. C.; Xavier, A. C.; Bianchi, T.; Oliveira, P. T.; Scanlon, B. R.; Lucas, M. C.; Wendland, E. (2015). Performance evaluation of rainfall estimates by TRMM Multisatellite Precipitation Analysis 3B42V6 and V7 over Brazil. Journal of Geophysical Research: Atmospheres, 120, 1-11, 28 Ago., 2015.

Morais, L. B. \& Ferreira, N. C. (2015). Banco de Dados Pluviométricos Integrados por Dados do Sensor TRMM e Estações Pluviométricas no Estado de Goiás, Anais XVII Simpósio Brasileiro de Sensoriamento Remoto - SBSR, João Pessoa-PB, Brasil, 25 a 29 de abril de 2015, INPE.

NASA, Giovanni User Guide; Giovanni is an online (Web) environment for the display and analysis of geophysical parameters in which the provenance (data lineage) can easily be accessed. Disponível em $<$ https://giovanni.gsfc.nasa.gov/giovanni/doc/UsersManualworkingdocument.docx.html $>$.

NASDA. (2001). TRMM Data Users Handbook. National Space Development Agency of Japan, Earth Observation Center.

Pereira, G., Silva, M. E. S., Moraes, E. C. \& Cardozo, F. S. (2013). Avaliação dos dados de precipitação estimados pelo satélite TRMM para o Brasil. RBRH: revista brasileira de recursos hídricos, v. 18, n. 3, p. 139-148.

Pombo, S.; Oliveira, R. P. (2015). Evaluation of extreme precipitation estimates from TRMM in Angola. Journal of Hydrology, 523, 663-679.

QGIS Development Team, (2018). QGIS Geographic Information System. Open Source Geospatial Foundation Project.

R CORE TEAM R; (2017). A Language and Enviorment for Statical Computing R Foundation, Vienna, Austria.

Soares, A. S. D., Paz, A. R. \& Piccilli, D. G. A. (2016). Avaliação das estimativas de chuva do satélite TRMM no Estado da Paraíba, Revista Brasileira de Recursos Hídricos - RBRH, 21(2). Versão On-line ISSN 2318-0331, Porto Alegre, abr./jun. 2016, p. 288 - 299, Artigo Científico/Técnico. 
Silva-Fuzzo, D. F. \& Rocha, J. V. (2016). Validação dos Dados de Precipitação Estimados Pelo TRMM, para o Estado do Paraná, e sua Contribuição ao Monitoramento Agrometeorológico. Revista Formação (ONLINE) Vol. 3; n. 23, p. 301316. ISSN: 2178-7298. ISSNL: $1517-543 X$.

Silva, M. I. C. A., Silva, R. M. \& Santos, C. A. G. (2016). Estimativa da precipitação do satélite TRMM para a Bacia do Alto Rio Paraíba (PB), XIII Simpósio de Recursos Hídricos do Nordeste.

Sodré, G. R. C., Borges, L. S., Oliveira, J. V. \& Mello, F. A. (2013). Análise Quantitativa e Qualitativa Horária de Dados do Radar, TRMM e Estações Meteorológicas na Região Amazônica. Revista Brasileira de Geografia Física, v. 6, p. 110-117. 